\title{
Clinical Data Management in Japan: Past, Present, and Future
}

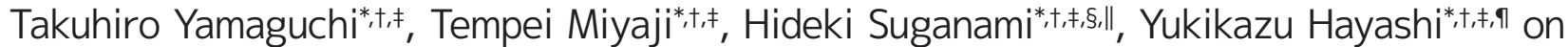 \\ behalf of SCDM Japan Steering Committee
}

In this article, we summarize 1) the history of clinical data management (CDM), 2) how different sectors function, 3) educational activities for CDM professionals, and 4) the role of data management in biomedical research in Japan. Here, we explain the differences in the role of clinical data managers in academic research organizations, pharmaceutical companies, and contract research organizations. The current status and issues of CDM in Japan are discussed, including training, education, and career paths for CDM professionals.

Keywords: Clinical data management; CDM; History; Japan

\section{History of CDM in Japan}

Until 1990, a function similar to clinical data management (CDM) was part of the monitoring department. Almost all clinical trials for new drug applications were conducted by pharmaceutical industries without outsourcing to contract research organizations (CROs) in Japan. A few biostatisticians, but no clinical data managers, were employed by almost all companies. The concept of data management (DM) was introduced in the field of clinical trials to Japan by Professor Yasuo Ohashi, the first representative director of the Japan Society of Clinical Trials and Research (JSCTR) in 1986. ${ }^{1}$ He shared his experience regarding a system of clinical trials in the coordinating center for the Lipid Research Clinics Program at North Carolina-Chapel Hill and Southwest Oncology Group (SWOG) Statistics and Data Management Center in Seattle. ${ }^{2}$ The implementation of the ICH-GCP ordinance in 1997 also had a major impact on clinical trials in Japan. As the quality of clinical trials in Japan has improved significantly, the cost of data management has also increased. The Japan Pharmaceutical Manufacturers Association (JPMA) started paying attention to DM, and in

\footnotetext{
* Division of Biostatistics, Tohoku University Graduate School of Medicine, JP

Department of Clinical Trial Data Management, Graduate School of Medicine, The University of Tokyo, JP

₹ Society for Clinical Data Management Japan Steering Committee, JP

§owa Company, Limited, JP

" Drug Evaluation Committee, Japan Pharmaceutical Manufacturers Association, JP

" A2 Healthcare Corporation, JP

Corresponding author: Tempei Miyaji, MSc

(tmiyaji@m.u-tokyo.ac.jp)
}

1998, the Drug Information Association (DIA) conducted a workshop on CDM, which has been held annually since then and is the oldest DIA workshop in Japan. In the field of investigator-initiated cancer research, the Statistical Center of Japan Clinical Oncology Group (JCOG), headed by Dr. Masanori Shimoyama, was established at the National Cancer Center in 1991 and has been a leading group in investigator-initiated clinical trials in Japan. ${ }^{3}$ In the early 1990s, several clinical societies published guidelines for clinical trials, and the establishment of data centers and DM departments were discussed as important topics.

Although the progress made since the 1990s has not been smooth, the last decade has seen regulatory authorities finally beginning to recognize the importance of CDM to support clinical trials and, therefore, include $\mathrm{CDM}$ in the requirements for infrastructure development. For example, under the leadership of Professor Ohashi, the Union of Japanese Scientists and Engineers (JUSE) launched an educational course for employees of pharmaceutical companies and CROs in 1999, but it neither initially met the needs of the participants nor supported the idea that data managers require a broad perspective. Today, seminars are designed for beginners and advanced data managers, ${ }^{4}$ with many participants from academia. Clinical trial design and analysis have improved due to greater understanding of these areas by researchers in clinical trials, as well as the contributions of biostatisticians. However, the technical aspects of CDM, such as data collection, entry, and checking, are still largely focused on DM in clinical trials. The level of understanding and the depth of skills in these areas vary among sites. The essence of CDM is to ensure that the data are of an appropriate quality for fair and scientific evaluation. This has not been recognized in many educational activities. 
The first textbook on CDM, Clinical Trial Data Management - Role and Importance of Data Management (in Japanese), was published by Professor Ohashi and Mr. Atsushi Tsujii in 2004. ${ }^{5}$ The Department of Clinical Trial Data Management, the first research department for CDM in Japan, was established at the University of Tokyo in 2007 to train data managers under a new education system that examines all relevant clinical trial processes to adapt to rapid changes in the medical environment as well as digitization and digitalization of clinical trials worldwide. ${ }^{6}$ At the same time, regulatory agencies initiated projects to improve clinical trial networks beginning in 2007. ${ }^{7}$ The Ministry of Health, Labor, and Welfare (MHLW) started a program for the development of infrastructure for clinical trials, while in parallel the Ministry of Education, Culture, Sports, Science, and Technology (MEXT) started a program for the development of translational research infrastructure. A MHLW and MEXT joint program called the "5-Year Action Plan for Clinical Trial Promotion" challenged the established processes in clinical trial activation, ${ }^{8}$ developing a vertical clinical trial network to conduct trials rapidly and efficiently. Core research hospitals were appointed by MHLW in a system similar to the Clinical and Translational Science Awards (CTSA) Program in the US. ${ }^{9}$ Additionally, the MHLW promoted the recruitment and training of clinical trial staff, such as clinical research coordinators (CRCs), biostatisticians, and CDMs. In 2007, MEXT initiated the Translational Research Network Program, which was taken over by the Japan Agency for Medical Research and Development (AMED) in $2017,{ }^{10}$ with the aim of fostering promising individuals from academia and strengthening infrastructure. In 2021, 10 universities were appointed as research support hubs. ${ }^{10}$ The government wanted to expand this model and registered major universities with particular research seeds. In 2015, AMED was established, and these two programs were incorporated into the project for translational and clinical research core centers. ${ }^{11}$ Currently, there are 14 clinical trial research centers that facilitate investigatorinitiated investigational new drug (IND)/first-in-human (FIH) studies. In this context, training and education of clinical trial support personnel, including data managers, has become an important issue. Table 1 shows the chronology of CDM history in Japan.

\section{How Work Varies across the Aforementioned Sectors}

Academic data managers have diverse roles; ${ }^{7,12}$ they not only take part in CDM, but also complete central monitoring and study management tasks on a daily basis. In addition, educating investigators and conducting research on CDM is an important task for academic data

Table 1: Chronology of CDM history in Japan.

\begin{tabular}{|c|c|}
\hline Year & Event \\
\hline 1986 & The concept of data management (DM) was introduced in the field of clinical trials by Professor Yasuo Ohashi \\
\hline 1994 & Japan CRO Association was established \\
\hline 1997 & Implementation of the ICH-GCP ordinance \\
\hline 1998-present & DIA Japan started an annual CDM workshop \\
\hline 2001 & A subcommittee of statistics and data management was developed in JPMA \\
\hline 2004 & $\begin{array}{l}\text { The first CDM textbook Clinical Trial Data Management - Role and Importance of Data Management } \\
\text { (in Japanese) was published by Professor Ohashi and Mr. Tsujii }\end{array}$ \\
\hline 2004-present & $\begin{array}{l}\text { First CDM seminar for industry and academia was organized by Professor Ohashi and Mr. Tujii with the sponsorship } \\
\text { of the JUSE }\end{array}$ \\
\hline 2005 & JPMA symposium "Challenges and prospects of EDC" was held \\
\hline 2007-present & The first research department of CDM was established at the University of Tokyo \\
\hline 2007-2012 & MHLW and MEXT joint program "5-year Action Plan for Clinical Trial Promotion" \\
\hline 2010 & Good Clinical Data Management Practices (2009 version) was partially translated into Japanese by JPMA \\
\hline 2012 & $\begin{array}{l}\text { Master's courses for CDM were established at the Division of Biostatistics, Tohoku University Graduate School of } \\
\text { Medicine }\end{array}$ \\
\hline 2015-2016 & CDM training seminars funded by National University Hospital Clinical Research Promotion Initiative (NUH-CRPI) \\
\hline 2015 & Japan Agency for Medical Research and Development (AMED) was established \\
\hline 2017-2018 & CDM training seminars funded by AMED \\
\hline 2017-present & $\begin{array}{l}\text { GCDMP Study Session started at Department of Clinical Trial Data Management, Graduate School of Medicine, } \\
\text { The University of Tokyo }\end{array}$ \\
\hline 2019-present & CDM training seminars funded by MHLW \\
\hline 2019-present & SCDM Japan Steering Committee was established \\
\hline 2020 & $\begin{array}{l}\text { Comprehensive education curriculum and self-learning management tools were developed based on the } \\
\text { GCDMP by AMED research project }\end{array}$ \\
\hline
\end{tabular}


managers as educators and researchers. Along with the diversification of data sources handled in clinical research and advances in research design, data collection, and analysis methods, the scope of work handled by academic data managers and the required skills have also changed significantly. In addition to applying scientific thinking skills to quality control in a wide range of operations, from protocol development to data collection and analysis, clinical data managers are required to develop data collection tools using efficient and reliable methods to build programs, such as data analysis software that can visualize the potential risks of the clinical research data, as well as to be able to effectively communicate with other professionals. There is a pertinent need for the development of educational programs that enable the acquisition of these skills as core competencies.

This situation is equivalent to that of data managers working at pharmaceutical companies and CROs, as described below. The pharmaceutical industry began to build DM sections in approximately 1990. In addition to the revision of the ICH-GCP, the requirements for data quality have increased. A subcommittee of statistics and data management was developed in 2001. This committee held a symposium, "Challenges and prospects of EDC," in 2005..$^{13}$ The role of CDM in pharmaceutical industries has expanded in many ways as technology and the environment have changed. As mentioned above, the essence of CDM is to ensure that data are of an optimum quality for fair and scientific evaluation. The roles of CDM include determining the method of data collection, defining the database, data cleaning, coding, managing devices for data collection, creating the database, and acting as a hub with other departments. Depending on the organization of each company, there are various ways to differentiate an organization by function or to develop multifunctional human resources. Recently, clinical data managers in industry have become the center of a quality management system for clinical trials and tackling the challenges of utilizing eSources (e.g., direct data capture (DDC) and electronic health records (EHR)).

Moreover, in CROs from the 1990s to the early 2000s, which saw substantial increases in trial sample sizes, the focus of CDM work was largely on the accurate conversion of paper case report form (CRF) data into electronic data. With the rise of electronic data capture (EDC), more emphasis has been placed on data cleaning, but clinical data managers are usually reluctant to hand over data until the data are fixed. In the late 2000s, with the increase in interim analyses, the opportunity to provide data during trials increased. The first textbook for interim analysis was published in 2004 by Muraoko et al. ${ }^{14}$ In addition, with the development of a risk-based approach (RBA), the need for timely access to data during the study increased. Recently, there has been an increasing demand for data collected from various devices to be immediately linked and integrated. The Japan CRO Association was established in 1994 with four companies and, as of July 2021, consists of 15 regular member companies and 30 supporting member companies, including system vendors. ${ }^{15}$

\section{Educational System of Clinical Data Management Professionals}

Although each organization should have a training curriculum, most organizations seem to rely mostly on on-the-job training (OJT). The first CDM seminar for industry and academia was organized by Prof. Ohashi and Mr. Tujii with the sponsorship of the JUSE in 2004. Since then, the seminar has been held annually, and more than 800 clinical data managers have attended this course. As of 2010, this seminar became a three-day course and was held online in 2021. Since 2015, a number of academic institutions have also held annual CDM training seminars funded by the National University Hospitals Clinical Research Promotion Institute (NUH-CRPI) (2015-16), AMED (2017-18), and MHLW (2019-present). In the fiscal year 2020, eight CDM training seminars were organized by a core clinical research hospital. ${ }^{16}$ Tohoku University held the first department that incorporated GCDMP into the curriculum of a postgraduate program and was the first graduate school to design a graduate course (master's and $\mathrm{PhD}$ ) for clinical research professionals, including clinical data managers. Master's courses for CDM were subsequently established at the Division of Biostatistics, Tohoku University Graduate School of Medicine in 2012 in order to train high-level clinical research administrators. ${ }^{12}$ The Department of Clinical Trial Data Management at the University of Tokyo Graduate School of Medicine has also held GCDMP study sessions for data managers since 2017, open to both industry and academia, and provided a forum for reading and discussing GCDMP content over the course of a year for free..$^{17}$ In 2020, AMED funded a year-long project to develop CDM education programs; ${ }^{18}$ in this project, a comprehensive education curriculum and self-learning management tools were developed based on the GCDMP and core competencies for CDM, which were identified based on a national survey in 2019.

There is no widely accepted or standardized certification program for CDM, which is one of the major challenges in CDM training. According to the SCDM website, approximately six Japanese people have obtained SCDM CCDM certification. ${ }^{19}$ Some of the reasons that few people in Japan have obtained the CCDM certificate may be the presence of a language barrier and the lack of recognition of CCDM in Japan. The SCDM Japan Steering Committee (JSC) was established in 2019, which aims to disseminate the value of CCDM to both industry and academia, enabling them to utilize it among employment criteria. ${ }^{20}$ Additionally, JSCTR is currently attempting to develop a CDM certification program; however, collaboration with the SCDM CCDM program is desirable, as it has become the global standard.

\section{Role of Data Management in Biomedical Research}

The MHLW's basic approach to the promotion of science and technology research through its policies is the realization of safe and quality healthcare. ${ }^{21}$ The approach consists of three elements: promotion of health safety, realization of advanced medical care, and security of health safety. Promotion of health safety includes research on the promotion of maternal and child healthcare, lifestyle 
diseases, mental health promotion, prevention, diagnosis and treatment methods for cancer, promotion of long-term care prevention, overcoming immune/allergic diseases, and improving quality of life for disabilities/intractable diseases. Realization of advanced medical care includes research on basic technologies for advanced medical care, promotion of the development of clinical trial/study foundations, research on practical applications of regenerative medicine, and research on health. Security of health safety includes research on emerging and re-emerging infectious diseases, health risk management measures, industrial safety, medical safety, harmonization/ assessment of regulations on drugs/medical devices, and food safety. These are promoted in accordance with the Science and Technology Basic Program of MEXT. ${ }^{22}$

In 2017, the Headquarters for the Promotion of Data Health Reform was established within the MHLW to conduct a wide range of cross-departmental studies. Data health reform refers to the MHLW's strategy to provide information and communication technology (ICT)-based health management and medical services and to build a platform that aggregates big data in the areas of health, medicine, and nursing care. ${ }^{23}$ With an aging population of $26 \%$, the highest in the world, Japan is faced with the need to develop an efficient healthcare delivery system, and the Data Health Reform Division aims to create an environment in which the public can efficiently receive healthcare services.

In such a situation, CDM is quickly and increasingly becoming a part of the scientific enterprise. In biomedical research, we believe that clinical data managers play a particularly important role in the following aspects: diversification of data sources, active use of smart trials, decentralized clinical trials (DCTs), eSource as ICT advances, and RWD, including public registries, such as the Clinical Innovation Network (CIN), ${ }^{24}$ and various databases, such as the National Database (NDB) $)^{25}$ and Medical Information Database Network (MID-NET). ${ }^{26}$ Changes in the environment of drugs, devices, and treatment development, such as the start of the industry-academia collaborative national cancer genome screening project (SCRUM-Japan), ${ }^{27}$ have led to more complex protocol designs, such as umbrella/basket study designs based on genomic biomarkers and adaptive studies. New data sources and new technologies used in clinical research are expected to continue to evolve, increasing the complexity of DM. Clinical data managers need to understand the changes that are underway and act in accordance with the essentials of CDM.

\section{Unique Strengths, Challenges, and Regulatory Issues}

One of the regulatory challenges is the existence of multiple forms of regulation in Japan, including the GCP ordinance, the Clinical Trials Act, ${ }^{28}$ and legal and ethical guidelines for research by various ministries. ${ }^{29}$ Different regulations apply depending on the type of trial and the presence of financial support for academic trials. Another specific challenge for clinical data managers is the lack of an established career path, especially in academia, in terms of stable contracts and salaries. As clinical data managers are not national or official qualifications, various models for careers in CDM need to be established and popularized. Although one qualification to show CDM professionality is obtaining SCDM CCDM certification, only a few CCDM holders exist in Japan, and certification remains unrecognized in industry and academia. Another challenge, as discussed above, is diversification of CDM tasks and roles. As CDM is evolving into clinical data science (CDS), clinical data managers need to acquire the knowledge and skills to deal with new streams and demands.

\section{Activities of SCDM Japan Steering Committee}

The Japanese branch of the Society for Clinical Data Management (SCDM) was officially approved in February 2019. SCDM JSC aims to 1) disseminate the activities of SCDM in Japan, 2) promote the value of CCDM among Japanese clinical data managers, 3) strengthen the CDM network through the SCDM Japanese regional office, and 4) provide a comprehensive educational program on CDM through SCDM educational materials, such as Good Clinical Data Management Practice (GCDMP), to clinical data managers in Japan. The Japanese webpage of the SCDM website(https://scdm.org/japan/) was published in August 2020. Sixteen steering committee members from academia, industry, and regulatory affairs manage SCDM Japan, and as of October 2021, more than 210 clinical data managers are registered in the network mailing list. Recently, the SCDM JSC conducted a survey to estimate the population of clinical data managers in Japan. In addition, JSC is now translating revised chapters and untranslated chapters of the GCDMP into Japanese. Deliverables of our project will be published on the SCDM website. It is expected that the activities of SCDM will contribute to the further development of CDM professionals and support CDM career path models in Japan.

\section{Conclusions}

In this article, we chronologically summarized the history of CDM in Japan. We also discussed the roles of CDM among different sectors, discussed the education system for clinical data managers, and introduced the activities of SCDM JSC. To the best of our knowledge, this is the first article to describe the history and status quo of CDM in Japan.

\begin{tabular}{|c|c|}
\hline \multicolumn{2}{|c|}{ Abbreviations } \\
\hline Abbreviation & Explanation \\
\hline$\overline{\mathrm{AMED}}$ & $\begin{array}{l}\text { The Japan Agency for Medical Research } \\
\text { and Development }\end{array}$ \\
\hline CCDM & Certified Clinical Data Manager \\
\hline CDM & Clinical Data Management \\
\hline CDS & Clinical Data Science \\
\hline CIN & The Clinical Innovation Network \\
\hline $\mathrm{CRC}$ & Clinical Research Coordinator \\
\hline $\mathrm{CRF}$ & Case Report Form \\
\hline CRO & Contract Research Organization \\
\hline CTSA & $\begin{array}{l}\text { The Clinical and Translational Science } \\
\text { Awards }\end{array}$ \\
\hline DCT & Decentralized Clinical Trial \\
\hline DDC & Direct Data Capture \\
\hline
\end{tabular}




$\begin{array}{ll}\text { DIA } & \text { Drug Information Association } \\ \text { DM } & \text { Data Management } \\ \text { EDC } & \text { Electronic Data Capture } \\ \text { EHR } & \text { Electronic Health Record } \\ \text { FIH } & \text { First-In-Human } \\ \text { GCDMP } & \text { Good Clinical Data Management Practice } \\ \text { ICH-GCP } & \text { The International Conference on } \\ & \text { Harmonization Good Clinical Practice } \\ \text { ICT } & \text { Information and Communication } \\ & \text { Technology } \\ \text { IND } & \text { Investigational New Drug } \\ \text { JCOG } & \text { Japan Clinical Oncology Group } \\ \text { JPMA } & \text { The Japan Pharmaceutical Manufacturers } \\ & \text { Association } \\ \text { JSC } & \text { Japan Steering Committee } \\ \text { JSCTR } & \text { Japan Society of Clinical Trials and Research } \\ \text { JUSE } & \text { The Union of Japanese Scientists and } \\ & \text { Engineers } \\ \text { MEXT } & \text { The Ministry of Education, Culture, } \\ & \text { Sports, Science, and Technology } \\ \text { MHLW } & \text { The Ministry of Health, Labor, and Welfare } \\ \text { MID-NET } & \text { Medical Information Database Network } \\ \text { NDB } & \text { National Database } \\ \text { NUH-CRPI } & \text { The National University Hospitals Clinical } \\ & \text { Research Promotion Institute } \\ \text { OJT } & \text { On-the-Job Training } \\ \text { RBA } & \text { Risk-Based Approach } \\ \text { SCDM } & \text { Society for Clinical Data Management } \\ \text { SWOG } & \text { Southwest Oncology Group } \\ & \end{array}$

\section{Acknowledgements}

We would like to express our sincere respect and appreciation to the late Professor Yasuo Ohashi for his great contribution to the establishment of a base and development of CDM in Japan. We would also like to thank Ms. Hisayo Ogawa for her assistance and Editage (http://www.editage.com) for editing and reviewing this manuscript for English language.

SCDM Japan Steering Committee Members: Reza Rostami (SCDM Board of Trusty Liaison; Duke Clinical Research Institute); Takuhiro Yamaguchi (Co-chairs; Tohoku University Graduate School of Medicine); Tempei Miyaji (Co-chairs; Graduate School of Medicine, The University of Tokyo); Hideki Suganami (Kowa Company, Limited); Yukikazu Hayashi (A2 Healthcare Corporation); Yoshiteru Chiba (University Hospital Medical Information Network Center); Koji Miyata (Astellas); Makoto Yokobori (Suxac Inc.); Hiroshi Ohtsu (Juntendo University); Masako Kurata (CHUGAI PHARMACEUTICAL CO., LTD); Yasuharu Shibata (MSD KK); Chiharu Shimada (Paraxel); Munenori Takata (Tohoku University Hospital); Satoru Fukimbara (Ono Pharmaceutical); Motohide Nishi (Medidata Solutions); Yuki Ando (Observer; Pharmaceuticals and Medical Devices Agency).

\section{Competing Interests}

Although article type is selected as "topics briefs and perspective", this article is intended to submit to "Special Issue on the Practice of Clinical Data Management Worldwide."

\section{References}

1. Ohashi Y. Clinical Data Management in Japan: Historical Perspectives and Future Challenges. 20th DIA Annual Workshop in Japan for Clinical Data Management; Tokyo, Japan; 2017.

2. Ohashi Y. Dr. Ohashi's Medical Statistics. Lifescience publishing; 2018. (in Japanese).

3. Japan Clinical Oncology Group. History. http:// www.jcog.jp/en/history/index.html.

4. Union of Japanese Scientists and Engineers (JUSE). Clinical Data Management Seminar https:// www.juse.or.jp/src/seminar/detail/99/26118 (in Japanese).

5. Ohashi Y, Tsujii A. Clinical trial data management - role and importance of data management. IGAKUSHOIN Ltd.; 2004(in Japanese).

6. Yamaguchi T. Establishment of a New Department of Clinical Trial Data Management (CTDM) in the University of Tokyo: Why We Discuss CTDM at This Time? 11th Annual Workshop in Japan for CLINICAL DATA MANAGEMENT; Tokyo, Japan 2008.

7. Miyaji T, Yamaguchi T. The Role of Academic Data Manager and Our Career Path: The Status Quo of Japanese Academic Setting. Oral presentation. SCDM 2016 Annual Conference; San Diego, CA 2016.

8. Ministry of Health, Labour and Welfare, Ministry of Education, Culture, Sports, Science and Technology. 5-Year Action Plan for Clinical Trial Promotion challenged for activation of clinical trials. https://www.mhlw.go.jp/shingi/2007/03/ s0330-5.html (in Japanese).

9. National Center for Advancing Translational Sciences. Clinical and Translational Science Awards (CTSA) Program. https://ncats.nih.gov/ctsa.

10. Japan Agency for Medical Research and Development. Translational Research Network Program. https://www.amed.go.jp/program/ list/16/01/009.html (in Japanese).

11. Japan Agency for Medical Research and Development. Overview of Department of Clinical Research and Trials. https://www.amed.go.jp/en/ program/list/05/index.html.

12. Yamaguchi T. Professional Training Course for Clinical Data Management in Tohoku University. SCDM 2017 Annual Conference; Orlando, FL 2017.

13. Japan Pharmaceutical Manufacturers Association Drug Evaluation Committee. Challenges and prospects of EDC. The 3rd Symposium of the Statistics and DM Section; Tokyo, Japan 2005 (in Japanese).

14. Muraoka R, Aoki Y, Takayama N, Suganami H, Koide T. Interim Analysis for Clinical Trials - From Basics to Implementation. Scientist Press Co., Ltd.; 2004 (in Japanese).

15. Japan CRO Association. Member List. https:// www.jcroa.or.jp/english/member.html.

16. Ministry of Health, Labour and Welfare. Data Manager Training Program. https://www.mhlw. go.jp/stf/newpage_11482.html (in Japanese). 
17. Department of Clinical Trial Data Management, Graduate School of Medicine, The University of Tokyo. GCDMP Study Session. http://ctdm.umin.jp/ news-list.html (in Japanese).

18. Japan Agency for Medical Research and Development. Central IRB Promotion Program. https://www.amed.go.jp/koubo/ 05/01/0501C_00130.html (in Japanese).

19. Society for Clinical Data Management. CURRENT CCDMS. https://scdm.org/current-ccdms/.

20. Society for Clinical Data Management Japan Steerring Committee. SCDM Japan Homepage. https://scdm.org/japan/ (in Japanese).

21. Ministry of Health, Labour and Welfare. Annual Health, Labour and Welfare Report 2020. https:// www.mhlw.go.jp/english/wp/wp-hw13/index. html.

22. Ministry of Education, Culture, Sports, Science and Technology. Science and Technology Basic Program. https://www.mext.go.jp/en/ policy/science_technology/lawandplan/title01/ detail01/1375311.htm.

23. Ministry of Health, Labour and Welfare. Data Health Reform Promotion Division. https://www.mhlw.go.jp/stf/seisakunitsuite/ bunya/0000148743.html.

24. The Clinical Innovation Network (CIN). https:// cinc.ncgm.go.jp/ (in Japanese).

25. Ministry of Health, Labour and Welfare. The National Database (NDB) https://www.mhlw.go.jp/ stf/seisakunitsuite/bunya/kenkou_iryou/iryouhoken/ reseputo/index_13898.html (in Japanese).

26. MID-NET (Medical Information Database Network) https://www.pmda.go.jp/safety/mid-net/0001. html (in Japanese).

27. National Cancer Center Japan. SCRUM-Japan GI-SCREEN Aims for Realizing of Cancer Precision Medicine Utilizing Liquid Biopsy by Analyzing Comprehensive Cancer Genome Alterations in Blood. https://www.ncc.go.jp/en/information/ press_release/20180313/index.html.

28. The Clinical Trials Act. https://www.mhlw.go.jp/ file/06-Seisakujouhou-10800000-Iseikyoku/ 0000213334.pdf.

29. Ministry of Health, Labour and Welfare. Research Guidelines. https://www.mhlw.go.jp/stf/ seisakunitsuite/bunya/hokabunya/kenkyujigyou/ikenkyu/index.html. (in Japanese).

How to cite this article: Yamaguchi T, Miyaji T, Suganami H, Hayashi Y, on behalf of SCDM Japan Steering Committee. Clinical Data Management in Japan: Past, Present, and Future. Journal of the Society for Clinical Data Management. 2021; 1(1): 13, pp. 1-6. DOI: https://doi.org/10.47912/jscdm.45

Copyright: (c) 2021 SCDM publishes JSCDM content in an open access manner under a Attribution-Non-Commercial-ShareAlike (CC BY-NC-SA) license. This license lets others remix, adapt, and build upon the work non-commercially, as long as they credit SCDM and the author and license their new creations under the identical terms. See https://creativecommons.org/licenses/by-nc-sa/4.0/. 